\title{
Design of a High Capacity Puck Storage System for Cryo-EM grids in a Facility Setting
}

\author{
Alberto Estevez ${ }^{1}$, Chris Arthur ${ }^{1}$, Alexis Rohou $^{1}$ and Claudio Ciferri ${ }^{1}$
}

1. Department of Structural Biology and Protein Chemistry, Genentech Inc. South San Francisco, California, United States of America.

Over the past few years Cryo-EM has undergone a resolution revolution due to important technical advances and the ability to obtain high-resolution structural information. Academia and Industry have focused their attention toward developing state of the art facilities and consortia to analyze samples and collect data.

With the introduction of automatic sample loaders and automatic data collection, it should be expected that the amount of samples stored and intended for viewing will increase as well. Historically, the number of users in a facility has been low and therefore low capacity storage systems have been sufficient. Currently, the most common method for storing Cryo-EM grids involves depositing several 4-slot grid boxes into a $50 \mathrm{ml}$ falcon tube. These tubes are then modified by lab personnel to have holes on the side, to allow for the entry of LN2, and also in the cap, for the addition of string, used for later retrieval of the tube from the storage container. Grids stored in this manner underutilize the capacity of the container, which now hosts a seemingly chaotic tangle of strings on the outside, each connected to a tube held within. Identification of the boxes inside are often arbitrarily coded by the person who stored them and very difficult to retrieve and organize. In a facility, where several personnel need to work together, having a standardized system to properly store grid samples is a must for correct sample tracking.

Many in the field have recognized the need to introduce new methods for dealing with the expected influx of prepared samples to be stored. Recently, a system has been described which uses a "puck" system modified from the type used for storing and shipping crystallography samples (G Scarpin, Workshop on the Management of Large CryoEM Facilities). This system describes the storage of 12 grid boxes into one puck, several pucks stacked on top of each other and inserted into a storage container. This type of system is more efficient than falcon tubes in its use of storage capacity. And, when combined with a standard log sheet, allows for a transparent method for tracking grids among many users. One limitation presented by this system though, is the ability to only handle complete pucks forcing the user to move 12 sample boxes at one time, even when interested in working on one specific grid box.

To improve on this, we have designed a 2-piece solution that works together to give a high capacity system with grid integrity and system capacity in mind. The first part consists of a custom-made grid box with 8 positions. This grid box will have the same dimensions and properties of commercially available grid boxes and could be manufactured on site using a 3D printer. This design would increase the number of grids that could be stored in a single grid box after vitrification and would represent an adequate number suitable for the Titan Krios autoloader cartridge. Manufacturing grid boxes in this way allows for different colors or customizable labeling that enhance storage tracking. The second part of the design consists of a custom made puck with 8 removable and independent subdivisions, each having enough room for one grid box. In this way, each of the 8 slices could be used independently, allowing 
the user to store or retrieve individual grid boxes, or they can be used in combination as a whole puck for shipping to a collaborator or consortium for data collection of several samples.

In summary, our system will allow for storage of grids within EM facilities and delivery of organized samples between different labs. A Dewar carrying 6 canes each comprised of six stacked pucks would allow for a projected capacity of $\sim 2300$ grids. We will implement this system in our facility as a standard method for grid storage and will make the design and system available for the Cryo-EM community. 